\title{
Relationship between SPOP mutation and breast cancer in Chinese population
}

\author{
M.A. Khan ${ }^{1 *}$, L. Zhu ${ }^{1 *}$, M. Tania ${ }^{1}$, X.L. $X_{i a o^{2}}$ and J.J. $F u^{1}$ \\ ${ }^{1}$ Key Laboratory of Epigenetics and Oncology, \\ The Research Center for Preclinical Medicine, \\ Sichuan Medical University, Luzhou, Sichuan, China \\ ${ }^{2}$ Department of Pathology, Affiliated Hospital of Sichuan Medical University, \\ Luzhou, Sichuan, China \\ *These authors contributed equally to this study. \\ Corresponding author: J.J. Fu \\ E-mail: fujunjiang@hotmail.com
}

Genet. Mol. Res. 14 (4): 12362-12366 (2015)

Received May 14, 2015

Accepted July 31, 2015

Published October 16, 2015

DOI http://dx.doi.org/10.4238/2015.October.16.2

\begin{abstract}
SPOP protein has been found to have ubiquitin ligase activity. Mutations in SPOP gene have been recently reported in some cancers such as prostate, gastric, colorectal cancer. We investigated SPOP DNA mutation in tumor tissues collected from 70 Chinese female breast cancer patients in Southwestern China by DNA sequencing. The results did not show mutation in our tissue samples, indicating that a mutation in the SPOP gene may not be associated with breast cancer, particularly in Chinese women. This DNA mutation analysis or DNA genotyping may provide useful and important information for genetic counseling and personalized medical treatment for different types of cancers.
\end{abstract}

Key words: Breast cancer; Chinese women; Exome sequencing; SPOP gene 


\section{INTRODUCTION}

Cancer is one of the major threats of modern life, of which one of the major causes is genetic abnormalities. In cancer cells, activated oncogenes promote cell growth, reproduction, and protection against programmed cell death. Another type of affected gene known as tumor suppressor gene is inactivated in cancer cells, resulting in a loss or decrease in its function, which inhibits cell division and survival. SPOP (speckle-type POZ protein) is a CULLIN3dependent ubiquitin ligase that contains an N-terminal TRAF-like domain and a C-terminal POZ domain. In humans, SPOP plays a conserved role in TNF-mediated JNK signaling and is highly expressed in $99 \%$ of clear cell renal cell carcinoma (CCRCC), and it has been suggested to be a specific tumor marker for RCC (Liu et al., 2009). A recent study reported that SPOP is one of the most frequently mutated genes in prostate cancer, at a frequency of $6-15 \%$ across localized and advance prostate tumors (Barbieri et al., 2012). SPOP functions as a tumor suppressor in prostate cells by promoting the turnover of steroid receptor coactivator-3 (SRC3) protein through its ubiquitination and proteasomal degradation and suppressing androgen receptor transcriptional activity. Prostate cancer-associated mutation in SPOP is reported to abrogate the tumor suppressor effect of SPOP (Geng et al., 2013). Another study by Kim et al. (2013) reported SPOP as a candidate tumor suppressor gene in prostate, gastric and colorectal cancers. Breast cancer is one of the most common cancers affecting women and a major women's health problem worldwide (Rezaeian et al., 2014; Khan et al., 2015). The incidence of breast cancer is rapidly increasing, especially in developing countries due to a lack of mass education and screening programs, poverty and poor access to health care facilities (Agarwal et al., 2009). American Cancer Society statistics showed that approximately 232,340 new cases of invasive breast cancer and 39,620 breast cancer deaths were expected to occur among US women in 2013 (DeSantis et al., 2014). For the development of breast cancer treatment, a clear understanding of breast carcinogenesis is important. To enrich our knowledge and information of genetic mutations related to breast cancer, we investigated in this study the possible mutations in the SPOP gene in breast tumor tissue of Chinese women.

\section{MATERIAL AND METHODS}

\section{Patient information}

Seventy female breast cancer patients were selected for this study from Sichuan Medical University affiliated hospital, which is located in Southwestern China. The age of the patients ranged from 26 to 67 . Cancer was pathologically confirmed in all patients, and more than half of the patients had confirmed metastasis. The study protocol was reviewed and approved by the Ethics Committee of Sichuan Medical College. All participants agreed to participate in the study and signed informed consent was obtained from each participant.

\section{Genomic DNA isolation from fresh tumor tissue}

Fresh biopsy samples of tumor tissues were collected from the cancer patients for DNA extraction. Tissue was homogenized and genomic DNA was extracted by using the TIANamp Genomic DNA kit (DP304-02, TIANGEN Biotech, Beijing, China). 


\section{Polymerase chain reaction}

DNA was then used for polymerase chain reaction (PCR) amplification of the 573-bp exome region spanning exon 5 , intron 5 and exon 6 , where the mutation hotspot is reported to be (Barbieri et al., 2013). The primers used for SPOP gene amplification were as follows: SPOP-G6: 5'-actccacttggggcttttc-3' and SPOP-G5: 5'-tccagttctatcaaaatggatgc-3'. The contents of the PCR system $(20 \mu \mathrm{L})$ were as follows: $2 \mu \mathrm{L}$ primer $(2.5 \mu \mathrm{M}), 3 \mu \mathrm{L}(30 \mathrm{ng})$ DNA template, $10 \mu \mathrm{L} 2 \mathrm{X}$ PCR Taq Mastermix (TianGen Biotech Co. Ltd., Beijing) and $5 \mu \mathrm{L}$ deionized water. PCR conditions were as follows: initial denaturation at $95^{\circ} \mathrm{C}$ for $90 \mathrm{~s}$, followed by 33 cycles of $40 \mathrm{~s}$ at $94^{\circ} \mathrm{C}, 60 \mathrm{~s}$ at $60^{\circ} \mathrm{C}$, and $40 \mathrm{~s}$ at $72^{\circ} \mathrm{C}$, and a final extension of $5 \mathrm{~min}$ at $72^{\circ} \mathrm{C}$. PCR of each accession was executed in an Applied Biosystems Veriti ${ }^{\circledR}$ 96-Well Thermal Cycler (Life Technology, USA). The PCR amplified products were separated by $1.5 \%$ agarose gel electrophoresis at $120 \mathrm{~V}$ for $45 \mathrm{~min}$, and DNA bands were visualized under UV light with a Universal Hood (Bio-Rad, Segrate, Italy). The amplified DNA portion was cut out and purified for sequencing by the Sanger method.

\section{Sequencing}

The amplified SPOP exome was then sequenced by using Sanger di-deoxy sequencing (Beijing ZiXi Biological Technology Co., Ltd., Bejing, China) with a specific primer, SPOP-G6 or SPOP-G5.

\section{RESULTS}

At total of 70 breast cancer tissue samples were collected from Chinese women in Southwestern China. DNA was extracted and amplified and the exome regions of the SPOP gene sequenced. Figure 1 shows a representative agarose gel electrophoresis image of the amplified SPOP exome, where the mutation hotspot is reported to be (Barbieri et al., 2012). Figure 2 shows the DNA sequencing result of a representative individual. No mutation was found in the exome of the SPOP gene in the breast cancer patients studied. Although SPOP mutation has been reported to be associated with some cancers, we did not find any mutation within the exome region of this gene in breast cancer tissue of 70 Chinese patients.

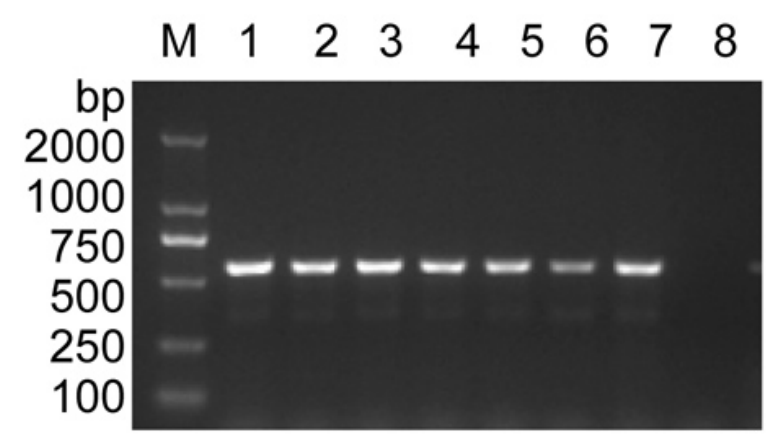

Figure 1. The agarose gel electrophoresis image of amplified SPOP exome. Lane $M=$ DL2000 DNA marker with indicated molecular weight size (bp). Lanes 1-7 = 573 bp of amplicons from seven tissue samples (TG04, TG05, TG17, TG18, TG40, TG46, TG147, TG23 and TG23 respectively). Lanes $8=$ negative control. 


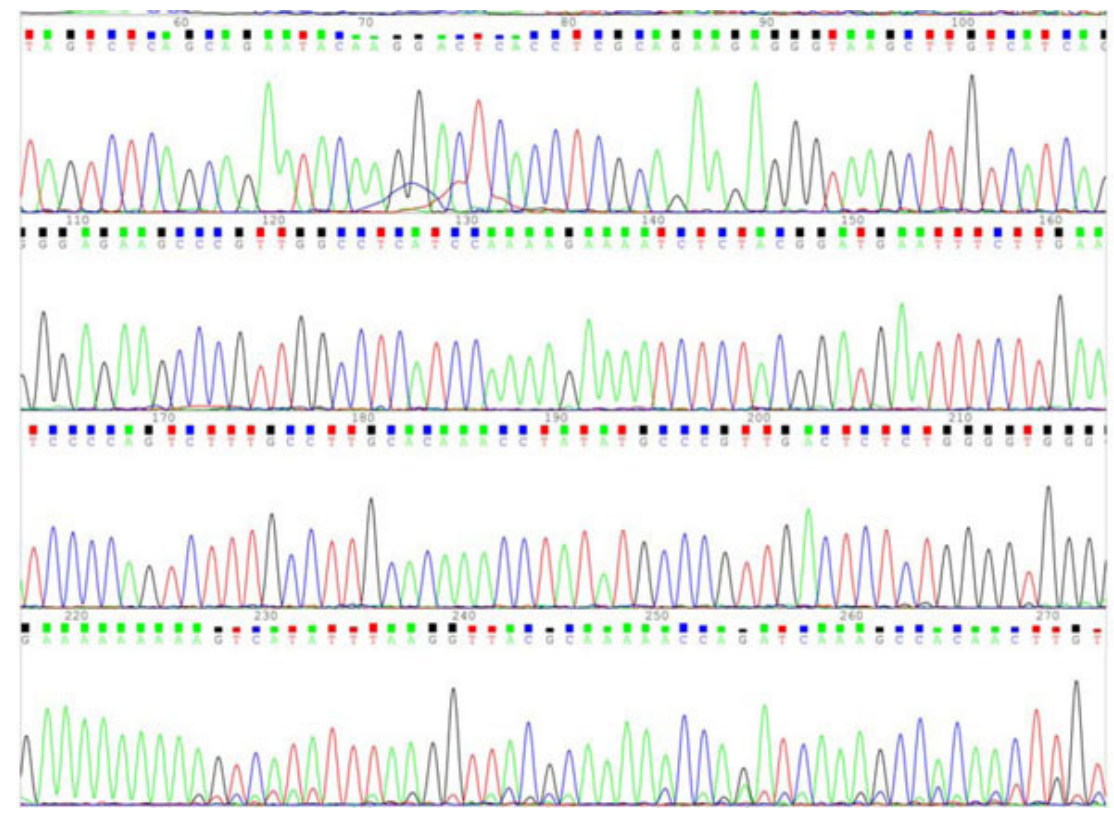

Figure 2. The partial DNA sequencing profiles of a representative individual. The DNA was cut and purified from agarose gel for Sanger sequencing.

\section{DISCUSSION}

Genetic alterations have been shown to play significant roles in carcinogenesis. The identification of genes involved in cancer development is critical for uncovering the molecular basis of cancer. Although genetic mutations are similar in every cancer type, some genes are specific to certain cancers. Mutations in BRCA1 and BRCA2 genes are the major genetic abnormalities reported in breast cancer, but mutations in some other genes such as P53, PTEN, STK11, CHEK2, ATM, BRIP1, PALB2, TWIST1 are also reported to be frequent (Gage et al., 2012; Khan et al., 2013). The identification of mutation in these genes has made the diagnosis of breast cancer comparatively easier, and has also led to some progress in understanding breast cancer and treatment development.

Comparatively, SPOP is a newly reported gene associated with cancer. It has been recently indicated as a tumor suppressor in cancers and demonstrated to have ubiquitin ligase activity (Li et al., 2011; Kim et al., 2011). In breast cancer, SPOP regulates breast cancer metastasis suppressor 1 (BRMS1) protein. SPOP acts as an adapter and mediates the interaction between cullin 3 (Cul3), a component of E3 ubiquitin ligase and BRMS1, and finally BRMS1 is destabilized by Cul3-SPOP (Kim et al., 2011). Before designing the study, we expected that a mutation in the SPOP gene may have an association with breast cancer. We sequenced the exome regions of the SPOP gene in the tumor tissues from seventy female breast cancer patients from Southwestern China. The pathological data from our tumor collection showed that most of the tumors were larger than $2 \mathrm{~cm}$ in diameter (mainly 2 to $5 \mathrm{~cm}$ ), which is bigger than that reported in Western countries. Thus, the samples we collected more likely represented late-stage breast tumors (Fu et al., 2012; Zhang et al., 2012). But interestingly, we still did not find any mutation in this gene. In some previous studies, mutations in SPOP exome regions have been reported to be associated with some cancer types, 
such as prostate, gastric and colorectal cancer (Barbieri et al., 2012; Geng et al., 2013; Kim et al., 2013). However, there is no reported study indicating the association of SPOP mutation with breast cancer. In this study, we found that mutation in the SPOP gene was not associated with breast cancer, particularly in Chinese women. Of course, it would be better if we could sequence the entire SPOP gene including regulatory regions to fully support our conclusion. A large-scale study with different ethnicities is recommended for the confirmation of an SPOP-breast cancer association. Also, mutations in SPOP in other cancer types should also be investigated, as this newly identified E3 ligase could be a potential tool in cancer therapeutics in the future. Therefore, DNA mutation analysis or DNA genotyping may provide useful and important information for genetic counseling and personalized medical treatment for different types of cancers.

\section{Conflicts of interest}

The authors declare no conflict of interest.

\section{ACKNOWLEDGMENTS}

Research supported by the Applied Basic Research Program of Science and Technology Department of Sichuan Province (\#14JC0797, \#2015JY0038) and Luzhou City Special Foundation (\#2013LZLY-J10), and in part by the National Natural Science Foundation of China (\#81172049), Science and Technology Innovation Team of Colleges and Universities of Sichuan Province (\#13TD0032).

\section{REFERENCES}

Agarwal G, Ramakant P, Forgach ER, Rendón JC, et al. (2009). Breast cancer care in developing countries. World J. Surg. 33: 2069-2076.

Barbieri CE, Baca SC, Lawrence MS, Demichelis F, et al. (2012). Exome sequencing identifies recurrent SPOP, FOXA1 and MED12 mutations in prostate cancer. Nat. Genet. 44: 685-689.

DeSantis C, Ma J, Bryan L and Jemal A (2014). Breast cancer statistics, 2013. CA Cancer J. Clin. 64: 52-62.

Fu J, Zhang L, He T, Xiao X, et al. (2012). TWIST represses estrogen receptor-alpha expression by recruiting the NuRD protein complex in breast cancer cells. Int. J. Biol. Sci. 8: 522-532.

Gage M, Wattendorf D and Henry LR (2012). Translational advances regarding hereditary breast cancer syndromes. J. Surg. Oncol. 105: 444-451.

Geng C, He B, Xu L, Barbieri CE, et al. (2013). Prostate cancer-associated mutations in speckle-type POZ protein (SPOP) regulate steroid receptor coactivator 3 protein turnover. Proc. Natl. Acad. Sci. U. S. A. 110: 6997-7002.

Khan MA, Chen HC, Zhang D and Fu J (2013). Twist: a molecular target in cancer therapeutics. Tumour Biol. $34: 2497-2506$.

Khan MA, Tania M, Wei C, Mei Z, et al. (2015). Thymoquinone inhibits cancer metastasis by downregulating TWIST1 expression to reduce epithelial to mesenchymal transition. Oncotarget 6: 19580-19591.

Kim B, Nam HJ, Pyo KE, Jang MJ, et al. (2011). Breast cancer metastasis suppressor 1 (BRMS1) is destabilized by the Cul3SPOP E3 ubiquitin ligase complex. Biochem. Biophys. Res. Commun. 415: 720-726.

Kim MS, Kim MS, Yoo NJ and Lee SH (2013). Mutational and expressional analyses of SPOP, a candidate tumor suppressor gene, in prostate, gastric and colorectal cancers. APMIS 121: 626-633.

Li C, Ao J, Fu J, Lee DF, et al. (2011). Tumor-suppressor role for the SPOP ubiquitin ligase in signal-dependent proteolysis of the oncogenic co-activator SRC-3/AIB1. Oncogene 30: 4350-4364.

Liu J, Ghanim M, Xue L, Brown CD, et al. (2009). Analysis of Drosophila segmentation network identifies a JNK pathway factor overexpressed in kidney cancer. Science 323: 1218-1222.

Rezaeian M, Sharifirad G, Mostafavi F, Moodi M, et al. (2014). The effects of breast cancer educational intervention on knowledge and health beliefs of women 40 years and older, Isfahan. Iran J. Educ. Health Promot. 3: 43.

Zhang L, Yang M, Gan L, He T, et al. (2012). DLX4 Upregulates TWIST and Enhances Tumor Migration, Invasion and Metastasis. Int. J. Biol. Sci. 8: 1178-1187. 